\title{
Aseptic zero discharge fluid dynamic gauging for measuring the thickness of soft layers on surfaces
}

\begin{tabular}{|c|c|}
\hline Journal: & Chemie Ingenieur Technik \\
\hline Manuscript ID & Draft \\
\hline Wiley - Manuscript type: & Kurzmitteilung \\
\hline Date Submitted by the Author: & $\mathrm{n} / \mathrm{a}$ \\
\hline Complete List of Authors: & $\begin{array}{l}\text { Wang, Shiyao; University of Cambridge, Department of Chemical } \\
\text { Engineering and Biotechnology } \\
\text { Schlüter, Florian; Technical University of Braunschweig, Institute for } \\
\text { Chemical and Thermal Process Engineering } \\
\text { Gottschalk, Nathalie; Technical University of Braunschweig, Institute for } \\
\text { Chemical and Thermal Process Engineering } \\
\text { Scholl, Stephan; Technical University of Braunschweig, Institute for } \\
\text { Chemical and Thermal Process Engineering } \\
\text { Wilson, D.; University of Cambridge, Department of Chemical Engineering } \\
\text { and Biotechnology } \\
\text { Augustin, Wolfgang; Technical University of Braunschweig, Institute for } \\
\text { Chemical and Thermal Process Engineering }\end{array}$ \\
\hline Keywords: & Biofouling, Dickenmessung, Rauigkeit, Analytik, Steriltechnik \\
\hline
\end{tabular}

\section{SCHOLARONE ${ }^{\text {m }}$}

Manuscripts 


\title{
Aseptic zero discharge fluid dynamic gauging for measuring the thickness of soft layers on surfaces
}

\section{Aseptisches Fluid Dynamic Gauging zur Messung der Dicke von weichen Schichten auf Oberflächen}

\author{
Shiyao Wang ${ }^{1}$, Florian Schlüter ${ }^{2}$, Nathalie Gottschalk ${ }^{2}$, Stephan Scholl ${ }^{2}$, D. Ian Wilson ${ }^{1}$ and \\ Wolfgang Augustin ${ }^{2, *}$ \\ ${ }^{1}$ Department of Chemical Engineering and Biotechnology, New Museums Site, Pembroke Street, \\ Cambridge, CB2 3RA, UK \\ ${ }^{2}$ Technische Universität Braunschweig, Institute for Chemical and Thermal Process Engineering, \\ Langer Kamp 7, Braunschweig, Germany \\ *Corresponding author: w.augustin@tu-braunschweig.de
}

\begin{abstract}
A bench-top device that can be used to perform fluid dynamic gauging measurements of soft solid layers with zero net liquid discharge and potentially under aseptic (closed system) conditions is demonstrated. Testing results are presented for Newtonian liquids with a range of viscosities: deionised water, $8 \mathrm{wt} \%$ and $10 \mathrm{wt} \%$ sucrose solutions, $18 \mathrm{wt} \%$ glycerol/water solutions and a paraffin oil (1.12 - $1000 \mathrm{mPa} \mathrm{s})$. The experimental data for discharge coefficient, $\mathrm{C}_{\mathrm{d}}$, against clearance/nozzle throat diameter, $\mathrm{h} / \mathrm{d}_{\mathrm{t}}$, gave good agreement with CFD simulations and the correlation presented by Tuladhar et al. [1]. The influence of surface roughness was studied by making measurements on a series of commercial sandpapers. The rough surfaces gave rise to systematic differences in $C_{d}$ from those obtained with smooth substrates which could not be corrected for using the height of the asperity peaks and careful calibration is therefore required when gauging rough surfaces.
\end{abstract}

Ein Laboranalysemethode zur Messung der Schichtdicke von weichen Feststoffablagerungen mit Hilfe des Fluid Dynamic Gauging wird vorgestellt. Dabei wird im Gegensatz zu bisherigen Anwendungen kein Fluid aus dem System entnommen, was einen aseptischen Betrieb ermöglicht. Erste Ergebnisse werden gezeigt für Newtonische Flüssigkeiten in einem weiten Viskositätsbereich: entionisiertes Wasser, 8 Gew.-\% and 10 Gew.-\% Zuckerlösung, 18 Gew.-\% Glyzerin/Wasser-Lösung und ein Paraffinöl (1,12 - $1000 \mathrm{mPa}$ s). Die experimentellen Daten für die Abhängigkeit des dimensionslosen Massenstrom $C_{d}$ vom Verhältnis Abstand/Düsendurchmesser $h / d_{t}$, zeigen eine gute Übereinstimmung mit CFD-Simulationen und der von Tuladhar et al. [1] präsentierten Korrelation. 
Der Einfluss der Oberflächenrauhigkeit wurde mit Hilfe verschiedene Sandpapiere untersucht. Es ergaben sich systematische Abweichungen von den Ergebnissen mit glatten Oberflächen, was nicht durch eine einfache Berücksichtigung der Rauheitsspitzen korrigiert werden konnte. Hier ist eine umfangreichere Kalibration erforderlich.

Key words: fluid dynamic gauging, aseptic, scanning, film thickness, surface roughness

\section{Introduction}

Biofilms, tissue cultures and proteinaceous fouling deposits are all examples of soft solid layers on surfaces. They are soft because they contain large amounts of liquid (i.e. high voidage) and the structural material has high elasticity or low strength. When removed from their natural (immersed) environment they often collapse. This creates problems when measuring the thickness of such layers, as non-contact techniques such as tomography are often expensive and require detailed calibration. Optical methods such as laser scanning confocal microscopy require the material or the liquid to be transparent to the light used.

The technique of fluid dynamic gauging (FDG) was developed by Tuladhar et al. [1] to allow the thickness of such layers to be measured in situ, in real time and relatively cheaply. Liquid is withdrawn or ejected, slowly, from a convergent nozzle located near the surface of the layer. The pressure drop and mass flow rate through the nozzle are measured, and together give an indication of how close the nozzle is to the layer surface. The nozzle location relative to the substrate is measured separately and the difference between the two distances gives the thickness of the layer. FDG does not require knowledge of physical and chemical properties of the solution and the layer (they can be opaque) except of the presence of a locally stiff surface. The forces exerted by the FDG flow can be estimated with confidence from computational fluid dynamics simulations (Chew et al. [2, 3]; Wang and Wilson [4]). The technique has been used to study the growth (fouling) and removal (cleaning) of a range of layer materials, and a scanning version of the device allowed different regions of a layer or substrate to be investigated (Gordon et al. [5]). A comprehensive description of the application spectrum is given in Augustin et al. [6].

In the early versions, the pressure drop across the nozzle was fixed and the flow rate measured. This required the use of significant volumes of liquid in an unsealed configuration. Given that many soft solid layers of interest would ideally be studied in a contained environment, e.g. for microbiological containment, and/or the inventory of liquid kept small, the technique of zero net discharge FDG (ZFDG) was developed. In this mode, the flow rate is set by a syringe pump and the pressure drop 
across the nozzle measured. The liquid is ejected and withdrawn from the test chamber in successive phases, so that no liquid enters or leaves the system. The operating principles of ZFDG were demonstrated by Yang et al. [7] and a system for studying layers on flat substrates was presented by Wang and Wilson [4]. Lemos et al. [8] bespoke ZFDG device to measure the thickness of Pseudomonas fluorescens biofilms on cylinders with a ZFDG unit.

The above ZFDG systems did not offer completely aseptic operation (small air channels were required to allow pressure equalisation). This paper describes the development of an aseptic ZFDG device which requires a modest volume of liquid (approximately two litres): the ability to use different liquids is demonstrated by calibration testing with liquids with a range of viscosities. The device features scanning capability and this is demonstrated in a short study of the effect of surface roughness on measurement. The latter studies also allowed the influence of surface roughness to be quantified, as layers of soft solids are sometimes uneven.

\section{ZFDG Apparatus}

ZFDG measurements are based around a convergent nozzle with throat diameter $d_{\mathrm{t}}$, through which liquid is withdrawn or ejected at a controlled rate by means of a syringe pump. The nozzle geometry and dimensions for this device are shown in Figure 1: its hydrodynamics in ejection and suction mode were studied experimentally and the results compared with computational fluid dynamics (CFD) simulations by Wang and Wilson [4].

The aseptic ZFDG test rig is similar to that used by Wang and Wilson [4] but features a smaller, (cylindrical) liquid reservoir, an $x-y$ stage which allows the substrate and layer being tested to be translated for scanning, and a method for isolating the contents from the surroundings. Figure 2 is a schematic of the apparatus while Figure 3 shows photographs of the system and key components. The reservoir (height $150 \mathrm{~mm}$, diameter $130 \mathrm{~mm}$, operating volume in these tests $=2 \mathrm{~L}$ ) was constructed from Perspex ${ }^{\mathrm{TM}}$ so that the layer could be monitored visually during testing.

Isolation is achieved by means of an aluminium foil (or flexible polypropylene film) which is secured to the top edge of the reservoir and to a ring on the nozzle mounting. The nozzle passes through a septum on the ring, providing a gas-tight seal. The airspace within the tent can be exchanged or purged as necessary. The flexibility of the film allows it to change shape as the liquid level changes when the gauging fluid is withdrawn or added to the reservoir via the nozzle, and adjusts with any transverse motion.

Liquid is fed or withdrawn by a computer controlled syringe pump (Hamilton ${ }^{\circledR}$ Glass, $d_{\mathrm{i}}=32.6 \mathrm{~mm}$ syringe; Harvard Apparatus PHD Ultra ${ }^{\mathrm{TM}}$ Series pump). The accuracy of the flow rate, $\dot{m}$, was 
measured as $1 \%$ of the set value. The nozzle (dimensions in Figure 1) was constructed from 304 stainless steel and is installed at the end of a long $(310 \mathrm{~mm})$ stainless steel tube. The nozzle is detachable so that different throat diameters and nozzle shapes can be employed as required. The nozzle vertical position is controlled by a stepper motor (Zaber Technologies, T-LSR075B, CE). Displacement in the horizontal plane in these tests was achieved by moving the reservoir and test stage using a manual $x-y$ stage (travel $95 \mathrm{~mm} \times 75 \mathrm{~mm}, \pm 0.01 \mathrm{~mm}$; KAMI, Germany): an automated stage would allow scanning as reported by Gordon et al. [5]. The nozzle vertical position (distance to the uncoated surface, labelled $h_{0}$ on Figure 1) is determined using feeler gauges with known thickness (e.g. $0.1 \mathrm{~mm}$ ). Calibration tests are usually started with $h_{0}=1.0 \mathrm{~mm}$.

The pressure drop across the nozzle, $\Delta P$, was measured by a pressure transducer (SensorTechnics HMAP001BU7H5) with an operating limit of approximately $7 \mathrm{kPa}$. Data collection and processing was performed with a LabVIEW ${ }^{\circledR}$ (National Instruments ${ }^{\mathrm{TM}}$ ) application, which also controlled the nozzle location and syringe pump motion. The LabVIEW code included a pressure difference cut-out to avoid damage to the pressure transducer.

\section{Material and Methods}

\subsection{Calibration}

The flow rate-pressure drop relationship is quantified in terms of the discharge coefficient, $C_{\mathrm{d}}$, which is the ratio of the actual to the ideal flow rates, viz.

$$
C_{d}=\frac{\dot{m}_{\text {actual }}}{\dot{m}_{\text {ideal }}}=\frac{4 \dot{m}}{\pi d_{t}^{2} \sqrt{2 \rho \Delta P}}
$$

where $\rho$ is the liquid density. The hydrodynamics of the flow through the nozzle are quantified in terms of the throat Reynolds number, defined

$$
R e_{\mathrm{t}}=4 \dot{m} / \pi \mu d_{\mathrm{t}}
$$

where $\mu$ is the liquid viscosity. For a given value of $R e_{\mathrm{t}}, C_{\mathrm{d}}$ is very sensitive to the clearance between the nozzle and the surface, labelled $h$ (with layer present) or $h_{0}$, for $h_{0} / d_{\mathrm{t}}<0.3$. In dimensionless terms,

$$
C_{d}=f\left(\frac{h_{0}}{d_{t}}, R e_{t}\right)
$$

For a given flow rate (and thus $R e_{\mathrm{t}}$ ), measurement of $\Delta P$ allows $C_{\mathrm{d}}$ to be evaluated and the distance of the nozzle from the test surface (uncoated substrate or layer) calculated. Tuladhar et al. [1] fixed $\Delta P$ and measured $\dot{m}$ : in ZFDG testing the reverse is done. 
Calibration tests were performed using clean, flat stainless steel substrates $(50 \mathrm{~mm}$ diameter, $3 \mathrm{~mm}$ thick). The nozzle, initially distant from the substrate, was moved towards the surface in steps of 0.1 $\mathrm{mm}, 0.05 \mathrm{~mm}$ and $0.02 \mathrm{~mm}$ as $h_{0}$ decreased. Liquid was alternately ejected then withdrawn at each nozzle location. The control software waited for the $\Delta P$ reading to reach steady state, which took about $4 \mathrm{~s}$. The pressure drop for the static (no flow) steady state was also measured so that any hydrostatic component arising from the difference in liquid levels could be accounted for. A feedback loop acted to stop and then withdraw the nozzle when the pressure drop approached the sensor's limit. In the tests presented here the nozzle-surface clearance reached approximately $0.1 \mathrm{~mm}$, subject to the above pressure drop criterion. Calibration plots $\left(C_{\mathrm{d}} v s h_{0} / d_{\mathrm{t}}\right)$ were then generated. Calibration tests obtained with the nozzle moving away from the substrate gave identical results.

Measured values of $C_{\mathrm{d}}$ are compared with values obtained from CFD simulations and a correlation based on experimental values, obtained for a similar nozzle with fixed $\Delta P$ and $\dot{m}$ measured, by Tuladhar et al. [1]. The simulations were performed using the COMSOL Multiphysics modelling platform. A detailed account of the computational methods and solution techniques is given in Wang and Wilson [4].

\subsection{Test liquids}

Calibration tests were performed with a range of Newtonian liquids: deionised water, paraffin oil, and solutions of glycerol and sucrose in deionised water. The physical properties of the gauging liquids are presented in Table 1 . Tests were performed at $16.5^{\circ} \mathrm{C}$ and atmospheric pressure. Flow rates of $5-$ $50 \mathrm{ml} / \mathrm{min}$ were used, giving $R e_{\mathrm{t}}$ values ranging from $0.36-943$. Only one flow rate, of $20 \mathrm{ml} / \mathrm{min}$, was used with $18 \mathrm{wt} \%$ glycerol and paraffin oil, the high viscosity of the latter giving $R e_{\mathrm{t}}=0.36$.

\subsection{Test surfaces}

The stainless steel disc was relatively smooth. Its arithmetic mean height of roughness, $R_{\mathrm{a}}$ (see Figure $4(c)$ ) of $0.2 \mu \mathrm{m}$ was considerably smaller than the smallest clearance used in ZFDG measurements (approximately $100 \mu \mathrm{m}$ ). The effect of surface roughness was investigated by gluing strips of sand paper $(3 \mathrm{M})$ of different grit sizes to a plate (see Figure $4(a)$ ) and generating calibration plots for each surface type in a series of tests. The roughness data reported by Mell [9] for similar materials are listed in Table 2. Deionised water was used as the gauging liquid and the nozzle was moved across the disc to make a series of measurements as shown in Figure 4(a).

On the smooth surface the nozzle location was zeroed by using feeler gauges of known thickness. The thickness of sandpaper, $\delta_{\mathrm{s}}$, was measured by a digital micrometer (Mitutoyo, Japan) with a stub diameter of $10 \mathrm{~mm}$, giving thickness ranging from $0.27 \mathrm{~mm}$ (P240) to $0.50 \mathrm{~mm}$ (P120). The initial estimate of the clearance, $h$, when performing calibration tests with sandpaper was calculated using 


$$
h=h_{0}-\delta_{s}
$$

Measuring $\Delta P$ for set values of $\dot{m}$ gave calibration plots of $C_{\mathrm{d}} v s h / d_{\mathrm{t}}$. The $h$ values were corrected to the true value of the nozzle - surface (rough) clearance, $h_{\text {true }}$, by (Figure 4(c))

$$
h_{\text {true }}=h+R_{p}
$$

[5]

where $R_{\mathrm{p}}$ is the average height between the peak and mean planes (see Table 2). Calibration plots show sets of $C_{\mathrm{d}} v s h_{\text {true }} / d_{\mathrm{t}}$.

\section{Results and Discussion}

\subsection{Reproducibility}

Reproducibility tests were performed using deionised water at a flow rate of $20 \mathrm{ml} / \mathrm{min}$ and $16.5^{\circ} \mathrm{C}$ $\left(R e_{\mathrm{t}}=377\right)$. The clean steel surface was gauged at a single point 10 times for each value of $h_{0}$ (from $0.5 \mathrm{~mm}$ to $0.1 \mathrm{~mm}$, with decrements of $0.1 \mathrm{~mm}$ ). The $C_{\mathrm{d}}$ values in Figure 5 show very good reproducibility, with standard error in both ejection and suction modes of less than $0.2 \%$. The region for ZFDG measurements, where $C_{\mathrm{d}}$ is usefully sensitive to $h_{0} / d_{\mathrm{t}}$, can be seen to lie between $0.05<$ $h_{0} / d_{\mathrm{t}}<0.25$, in agreement with Yang et al. [7] and Wang and Wilson [4]. At higher values of $h_{0} / d_{\mathrm{t}}, C_{\mathrm{d}}$ reaches an asymptote (in this case $\sim 0.8$, depending on the imposed flowrate). The agreement with CFD predictions is good.

The main uncertainties in measurements arose from the accuracy of zeroing the nozzle-substrate clearance, estimated at $\pm 5 \mu \mathrm{m}$, since $\Delta P$ was very sensitive to lower values of $h$. This was minimised by using different feeler gauges to crosscheck the initial clearance. The accuracy of the mass flow measurements was good (around $1 \%$ ). The pressure transducer uncertainty was reduced by increasing the signal to noise ratio using Fast Fourier Transform (FFT) analysis.

\subsection{Different gauging liquids}

Figure 6 shows calibration plots for the different liquids in Table 1 for $(a)$ ejection and $(b)$ suction, respectively, at a flowrate of $20 \mathrm{ml} / \mathrm{min}$. Suction mode gives lower $C_{\mathrm{d}}$ values than ejection, which is due to a higher pressure drop through the nozzle at these Reynolds numbers associated with the presence of a recirculation zone downstream of the nozzle throat (see Chew et al. [2]). The error bar for each datum is dominated by the uncertainty in the pressure measurement and these are smallest at low clearance: there is thus higher reliability in the pseudo linear region (see insets). Also shown on the plot are the results from CFD simulations for deionised water in both ejection and suction modes. The predictions again give good agreement, within experimental uncertainty.

The results for solutions with low viscosity, namely water and the sucrose solutions, exhibit a common trend of a pseudo-linear region $\left(0.1<h_{0} / d_{\mathrm{t}}<0.24\right)$ followed by an approach to an asymptote 
at large $h_{0} / d_{\mathrm{t}}$. With the more viscous liquids, namely $18 \%$ glycerol solution and the paraffin, the contribution from viscous dissipation dominates that from inertia: smaller $C_{\mathrm{d}}$ values are obtained and the transition is more gradual. The ideal flow term in the definition of $C_{\mathrm{d}}$, Equation (1), is based on an inertial result, so $C_{\mathrm{d}}$ is not expected to describe the pressure drop at low $R e_{\mathrm{t}}$ well. This was discussed at length by Chew et al. [3]. For ZFDG testing, the overall $\Delta P$ increases slowly as the nozzle approaches the substrate and the usefully linear region is hard to achieve with viscous gauging liquids. This result, combined with the potential for disruption of the layer caused by the high shear stress imposed by the flow on the surface, means that highly viscous liquids are less well suited for ZFDG applications.

\subsection{Effect of gauging flow rate}

The effect of gauging flow rate (and $R e_{\mathrm{t}}$ ) on $C_{\mathrm{d}}$ was investigated for the less viscous liquids. The influence of flow rate has been studied previously for 'mass flow' FDG, where the pressure drop (hydrostatic head) is controlled and the flow rate measured, by Tuladhar et al. [1] and Chew et al. [2, 3]). The objective of this work was to establish whether similar effects are seen in 'pressure mode' measurements, where the flow rate is controlled. The results in Figure 7 show similar trends to those noted above: $C_{\mathrm{d}}$ was generally larger for ejection than suction; $C_{\mathrm{d}}$ increases with $h_{0} / d_{\mathrm{t}}$, and, in the usefully linear region, increases weakly with $R e_{\mathrm{t}}$. In the asymptotic region (Figure $7(\mathrm{a})$ ), $C_{\mathrm{d}}$ is approximately 0.8 for all $R e_{\mathrm{t}}$. These observations are consistent with the findings of Chew et al. [2]. Figure 7(b-d) shows that for smaller $h_{0} / d_{\mathrm{t}}$ values, the effect of viscosity is not accounted for by $R e_{\mathrm{t}}$, as postulated in Equation (3). This is consistent with the influence of viscous dissipation and the use of an inertial loss term in defining $C_{\mathrm{d}}$, as noted above. The data obtained for smaller flowrates, such as 5 $\mathrm{ml} / \mathrm{min}$, are less consistent but agree within the experimental uncertainty which arises from the sensitivity of the pressure measurements.

The agreement with 'mass flow' FDG was investigated further by comparing the results obtained in suction mode with the correlation for $C_{\mathrm{d}}$ obtained by Tuladhar et al. [1] from experimental data collected over the range $400<R e_{\mathrm{t}}<2000$ for a nozzle similar to that in Figure 1. Its dimensions were: $d_{\mathrm{t}}=1.0 \mathrm{~mm}, d_{\mathrm{i}}=4.0 \mathrm{~mm}, w_{\mathrm{r}}=0.5 \mathrm{~mm}$ and $w_{\mathrm{e}}=0.1 \mathrm{~mm}$. They reported

$1000 C_{d}=\left(0.3571 \exp \left[-5.0613 \sqrt{\frac{h_{0}}{d_{t}}}\right]\right) R e_{t}+\left(-70.3+3721.2\left(\frac{h_{0}}{d_{t}}\right)-2238.3\left(\frac{h_{0}}{d_{t}}\right)^{2}\right)$

The results from the present work, for $h_{0} / d_{\mathrm{t}}=0.1$ and 0.14 , are plotted alongside Tuladhar et al.'s data in Figure 8: there are excellent agreements for the water values. There is also good agreement with the CFD simulation results for deionized water over the range $90<R e_{\mathrm{t}}<1200$. For a given value of $h_{0} / d_{\mathrm{t}}$ Equation (6) predicts a linear relationship, viz.

$$
h_{0} / d_{\mathrm{t}}=0.14 \quad C_{d}=5.37 \times 10^{-5} R e_{t}+0.41
$$




$$
h_{0} / d_{\mathrm{t}}=0.10 \quad C_{d}=7.20 \times 10^{-5} R e_{t}+0.28
$$

The Figure shows reasonably good agreement with the water data for $R e_{\mathrm{t}}>300$. There is poorer agreement for lower $R e_{\mathrm{t}}$ and for the viscous liquids.

The CFD simulations have been performed for the current nozzle geometry, i.e. $d_{\mathrm{t}}=1.0 \mathrm{~mm}, d_{\mathrm{i}}=5.9$ $\mathrm{mm}, w_{\mathrm{e}}=0.15 \mathrm{~mm}, w_{\mathrm{r}}=1.0 \mathrm{~mm}$ and $\theta=45^{\circ}$. By applying the simulation protocol proposed by Wang and Wilson [4], $C_{\mathrm{d}}$ for both ejection and suction modes were extracted and the empirical correlation (Equation (3)) was investigated. $C_{\mathrm{d}}$ is a relatively weak function of $\ln \left(R e_{\mathrm{t}}\right)$ within the useful measurement region, $0.1<h_{0} / d_{\mathrm{t}}<0.24$, viz.

$$
\begin{aligned}
& C_{d, E}=\left(0.036+1.093\left(\frac{h_{0}}{d_{t}}\right)-5.571\left(\frac{h_{0}}{d_{t}}\right)^{2}\right) \ln \left(R e_{t}\right)+\left(-0.689+5.094\left(\frac{h_{0}}{d_{t}}\right)+0.576\left(\frac{h_{0}}{d_{t}}\right)^{2}\right) \\
& C_{d, S}=\left(0.068-0.134\left(\frac{h_{0}}{d_{t}}\right)+0.105\left(\frac{h_{0}}{d_{t}}\right)^{2}\right) \ln \left(R e_{t}\right)+\left(-0.54+6.49\left(\frac{h_{0}}{d_{t}}\right)-13.509\left(\frac{h_{0}}{d_{t}}\right)^{2}\right)
\end{aligned}
$$

This model effectively corrects Tuladhar's [1] model for the lower range of $R e_{\mathrm{t}}$, especially from 90 to 400 and agrees with larger $R e_{\mathrm{t}}$ above 400 in the laminar flow regime.

There is thus good agreement between the two modes of FDG measurement. ZFDG offers robust and reliable operation while offering the capacity to use small liquid inventory and potentially hazardous substances.

\subsection{Effect of surface roughness}

The smooth and sandpaper surfaces were studied using deionised water at $16.5^{\circ} \mathrm{C}$ as the gauging liquid with flow rates ranging from $10-40 \mathrm{ml} / \mathrm{min}$, corresponding to $R e_{\mathrm{t}}=189-754$. Surface roughness had no effect at large clearance values. Examples of results are presented for $h / d_{\mathrm{t}}=0.10$ (of interest for gauging measurements) for both ejection and suction modes in Figure 9.

For both smooth and rough surfaces, $C_{\mathrm{d}}$ increases as flowrate and clearance increases, which agrees with Equation (6). For a given combination of $R e_{\mathrm{t}}$ and $h / d_{\mathrm{t}}, C_{\mathrm{d}}$ is larger for a rougher surface. This is due to the systematic effect of roughness elements on the estimated clearance, presented in Figure 4(c). For rougher surfaces, such as P120, the peak to trough region is available for liquid flow so the true thickness of the flow region is larger than the set value (equation (5)): $h_{0} / d_{\mathrm{t}}$ is therefore larger than $h / d_{\mathrm{t}}$, and $C_{\mathrm{d}}$ is consequently greater.

The values for P150 and P180 lie within the measurement uncertainty. The difference in roughness of two surfaces is $\sim 0.5 \mu \mathrm{m}$, which is lower than the resolution $(\sim 5 \mu \mathrm{m})$ of the gauging technique.

Figure 9 indicates that roughness elements of the size and nature found on sandpapers introduce significant uncertainty in the ZFDG measurements. One method for correcting for the known 
roughness based on peak heights is shown in Figure 4(c), yielding Equation (5). Figure 10 shows series of corrected data sets presented as plots of $C_{\mathrm{d}} v s h_{\text {true }} / d_{\mathrm{t}}$. Data for two flowrates are presented: seven flow rates were tested. The correction protocol gives good agreement for ejection at higher flowrates, i.e. $500<R e_{\mathrm{t}}<754$, but for lower ejection flow rates and in all suction mode tests $C_{\mathrm{d}}$ is systematically underestimated. The correction protocol gives good agreement between the rough surfaces, but differs from the smooth values by up to 0.1 . This difference is significant for measurements of absolute layer thickness. The good agreement between corrected values indicates that the correction protocol needs refinement. The peak height calculation gives an approximate account for the difference in flow area: the increase in surface area of the substrate, which will increase $\Delta P$ and reduce $C_{\mathrm{d}}$, has not been included._In the absence of a more reliable correction protocol, careful calibration using surfaces of similar roughness would be needed in order to make measurements of such layers.

\section{Conclusions}

\section{$\mid$}

The technique of fluid dynamic gauging was extended for the use with zero net liquid discharge and potentially under aseptic conditions. A new measuring device was designed and manufactured. Proofof-concept results have been obtained for a series of Newtonian liquids, ranging in viscosity from water to paraffin oil. The discharge coefficient is usefully sensitive to the nozzle-substrate clearance in the range $0.05<h_{0} / d_{\mathrm{t}}<0.25$ for all but the most viscous liquid, for which the sensitivity is poorer. These results showed good agreement with computational fluid dynamics simulations and, within the range of testing, the correlation presented by Tuladhar et al. [1].

Testing on surfaces with mixed roughness profiles demonstrated that the device can be used in scanning mode. Data obtained with a series of commercial sandpapers indicated that surface roughness can have a significant effect on ZFDG measurements, which could not be accounted for by a simple peak-to-height correction. Careful calibration is therefore required when making measurements on such surfaces.

\section{Acknowledgements}

Development of the ZFDG concept was supported by the Royal Society’s Paul Instrument Fund. The test rig was constructed by the technical team, in particular Karl Karrenführer, Jörg Leppelt and Sven Lorenzen, at the Institute for Chemical and Thermal Process Engineering, Technische Universität Braunschweig. Funding from Fitzwilliam College for Shiyao Wang is also gratefully acknowledged. 


\section{Symbols used}

\begin{tabular}{|c|c|c|}
\hline$C_{\mathrm{d}}$ & {$[-]$} & Fluid discharge coefficient \\
\hline$d_{\mathrm{i}}$ & {$[\mathrm{m}]$} & Inner diameter \\
\hline$d_{\mathrm{t}}$ & {$[\mathrm{m}]$} & Diameter of nozzle throat \\
\hline$h_{\text {true }}$ & {$[\mathrm{m}]$} & True initial gauging clearance \\
\hline$h_{0}$ & {$[\mathrm{~m}]$} & Nozzle-substrate clearance \\
\hline i.d. & {$[\mathrm{m}]$} & Internal diameter \\
\hline$\dot{m}$ & {$\left[\mathrm{~kg} \cdot \mathrm{s}^{-1}\right]$} & Mass flow rate \\
\hline$\Delta P$ & {$\left[\mathrm{~kg} \cdot \mathrm{m}^{-1} \cdot \mathrm{s}^{-2}\right]$} & Differential pressure $\left(\Delta P_{\mathrm{dyn}}-\Delta P_{\text {static }}\right)$ \\
\hline$\Delta P_{\mathrm{dyn}}$ & {$\left[\mathrm{kg} \cdot \mathrm{m}^{-1} \cdot \mathrm{s}^{-2}\right]$} & Dynamic pressure measurement \\
\hline$\Delta P_{\text {static }}$ & {$\left[\mathrm{kg} \cdot \mathrm{m}^{-1} \cdot \mathrm{s}^{-2}\right]$} & Static pressure measurement \\
\hline$R_{\mathrm{a}}$ & {$[\mathrm{m}]$} & Arithmetical mean height \\
\hline$R e_{\mathrm{t}}$ & {$[-]$} & Reynolds number at nozzle throat \\
\hline$R_{\mathrm{p}}$ & {$[\mathrm{m}]$} & Average height between highest peak and mean plane \\
\hline$R_{\mathrm{z}}$ & {$[\mathrm{m}]$} & Average height between highest peak and deepest valley \\
\hline$w_{\mathrm{e}}$ & {$[\mathrm{m}]$} & Nozzle rim width \\
\hline$w_{\mathrm{r}}$ & {$[\mathrm{m}]$} & Nozzle rim thickness \\
\hline Greek & & \\
\hline$\delta_{\text {true }}$ & {$[\mathrm{m}]$} & True thickness of sandpaper \\
\hline$\delta_{\mathrm{s}}$ & {$[\mathrm{m}]$} & Thickness of sand paper measured by Micrometer \\
\hline$\Phi$ & {$[\mathrm{m}]$} & Diameter \\
\hline$\varepsilon$ & {$[-]$} & Uncertainty \\
\hline$\mu$ & {$\left[\mathrm{kg} \cdot \mathrm{m}^{-1} \cdot \mathrm{s}^{-1}\right]$} & Fluid viscosity \\
\hline$\rho$ & {$\left[\mathrm{kg} \cdot \mathrm{m}^{-3}\right]$} & Fluid density \\
\hline Acronym & & \\
\hline CFD & \multicolumn{2}{|c|}{ Computational fluid dynamics } \\
\hline DAQ & \multicolumn{2}{|c|}{ Data acquisition } \\
\hline FFT & \multicolumn{2}{|c|}{ Fast Fourier Transform } \\
\hline LabVIEW & \multicolumn{2}{|c|}{ Laboratory virtual instrument engineering workbench } \\
\hline PT & \multicolumn{2}{|c|}{ Pressure transducer } \\
\hline SS & \multicolumn{2}{|c|}{ Stainless Steel } \\
\hline ZFDG & \multicolumn{2}{|c|}{ Zero (net) discharging fluid dynamic gauging } \\
\hline
\end{tabular}




\section{References}

[1] T.R. Tuladhar, W.R. Paterson, N. Macleod, and D.I. Wilson. The Can. J. Chem. Eng. 2000, 78(October). DOI:10.1002/cjce.5450780511

[2] J.Y.M. Chew, S.S.S. Cardoso, W.R. Paterson, and D.I. Wilson, Chem. Eng. J. 2004a, 59(16). DOI:10.1016/j.ces.2004.03.042

[3] J.Y.M. Chew, W.R. Paterson, and D.I. Wilson, J. Food Eng. 2004b, 65(2). DOI:10.1016/j.jfoodeng.2004.01.013

[4] S. Wang \& D.I. Wilson, Ind. Eng. Chem. Res. 2015, 54. DOI: 10.1021/acs.iecr.5b01956

[5] P.W. Gordon, A.D.M. Brooker, J.Y.M. Chew, D.I. Wilson, and D.W. York, Measurement Sci. Tech. 2010, 21(8). DOI: 10.1088/0957-0233/21/8/085103

[6] W. Augustin, Y.M.J. Chew, P.W. Gordon, V.Y. Lister, M. Mayer, W.R. Paterson, J.M. Peralta, S. Scholl, and D.I. Wilson, Chem. Ing. Tech. 2012, 84(1). DOI: 10.1002/cite.201100165

[7] Q. Yang, A. Ali, L. Shi and D.I. Wilson, J. Food Eng. 2014, 127. DOI: 10.1016/j.jfoodeng.2013.11.024

[8] M. Lemos, S. Wang, A. Ali, M. Simões, and D.I. Wilson, Biochem. Eng. J. 2016, 106. DOI: 10.1016/j.bej.2015.11.006

[9] B. Mell, 2010. Sandpaper Roughness Measurement using 3D Profilometry. NANOVEA ${ }^{\circledR}$ 


\section{Tables}

Table 1. Physical properties of gauging liquids $\left(16.5^{\circ} \mathrm{C}\right)$

\begin{tabular}{lccccc}
\hline Gauging liquid & $\rho$ & $\mu$ & $R e_{\mathrm{t}}(20 \mathrm{ml} / \mathrm{min})$ & Supplier & Symbol \\
& $\mathrm{kg} / \mathrm{m}^{3}$ & $\mathrm{mPa} . \mathrm{s}$ & - & & Figure 8 \\
\hline Deionised water & 997.3 & 1.12 & 377 & $\mathrm{~N} / \mathrm{A}$ & $\mathbf{\square}$ \\
$8 \mathrm{wt} \%$ sucrose & 1029.9 & 1.25 & 349 & Sigma & $\boldsymbol{\Delta}$ \\
$10 \mathrm{wt} \%$ surcrose & 1038.1 & 1.33 & 331 & Sigma & $\bullet$ \\
$18 \mathrm{wt} \%$ glycerol & 1047.6 & 1.65 & 250 & Aldrich & N/A \\
Paraffin oil & 860 & 1000 & 0.36 & Sigma-Aldrich & N/A \\
\hline
\end{tabular}

Table 2. Sandpaper surface roughness measures, see Figure 4(b): $R_{\mathrm{a}}$ (arithmetical mean height), $R_{\mathrm{z}}$ (average height between highest peak and deepest valley), $R_{\mathrm{p}}$ (average height between highest peak and mean plane), and average particle sizes

\begin{tabular}{cccccc}
\hline Surface & $R_{\mathrm{a}}$ & $R_{\mathrm{z}}$ & $R_{\mathrm{p}}$ & $\begin{array}{c}\text { Average particle diameter } \\
\text { Symbol }\end{array}$ & Figure 10 \\
& $\mu \mathrm{m}$ & $\mu \mathrm{m}$ & $\mathrm{mm}$ & $\mu \mathrm{m}$ & Lines \\
\hline Smooth & 0.2 & 0.97 & 0.0007 & N/A & $\diamond$ \\
P240 & $\sim 14.26$ & $\sim 169.6$ & 0.12 & 68 & $\Delta$ \\
P180 & 17.43 & 198.1 & 0.14 & 82 & $\square$ \\
P150 & 16.89 & 229.9 & 0.17 & $92-100$ & 0 \\
P120 & 24.41 & 273.2 & 0.20 & $115-125$ & $\square$ \\
\hline * the data are for P220 due to lack of data on P240 &
\end{tabular}

* the data are for P220 due to lack of data on P240 


\section{Figure legends}

Figure 1. Schematic of ZFDG nozzle geometry. $h_{0}$ is the clearance between the nozzle and the substrate. Dimensions: $\theta=45^{\circ}, d_{\mathrm{t}}=1.0 \mathrm{~mm}, d_{\mathrm{i}}=5.9 \mathrm{~mm}, w_{\mathrm{e}}=0.15 \mathrm{~mm}, w_{\mathrm{r}}=1.0 \mathrm{~mm}$. Dotted streamline indicates the flow path taken by the liquid in the ejection phase; the dashed streamline shows that in suction (with flow recirculation which occurs under certain operating conditions)

Figure 2. Schematic of aseptic ZFDG system

Figure 3. Photographs of ZFDG (a) test rig, and (b) detailed view of $1 \mathrm{~mm}$ i.d. nozzle and substrate. Labels: A, linear slide; B, pressure transducer; C, gauging tank; D, $x-y$ table

Figure 4. (a) Photograph, plan view of stainless steel disc with strips of sandpaper of different grit sizes attached. The gauging nozzle was moved across trajectory AA. Points indicate where gauging measurements were made; $(b)$ Side view (not to scale) schematic of sandpaper thicknesses for zeroing, $\delta_{1-4}-$ thicknesses of sandpaper (P180, P150, P240 and P120, respectively); (c) Schematic (not to scale) of rough surface: $R_{\mathrm{p}}$ is the average height between highest peak and mean plane, $R_{\mathrm{a}}$ is the mean roughness, $\delta_{\mathrm{s}}$ is the thickness measured by micrometer, $\delta_{\text {true }}$ the true thickness, $h_{\text {true }}$ the corrected/true clearance from the nozzle to mean plane of sandpaper. The smooth substrate is the reference plane

Figure 5. Reproducibility of ZFDG measurements on clean steel substrate. Deionised water at $16.5^{\circ} \mathrm{C}$, flow rate $20 \mathrm{ml} / \mathrm{min}$. Ten data sets were collected at each $h_{0} / d_{\mathrm{t}}$ value from 0.1 to 0.5 . The mean $C_{\mathrm{d}}$ value is plotted against $h_{0} / d_{\mathrm{t}}$ : the standard deviation is less than $0.2 \%$ of each datum (not shown), and uncertainty in $h$ is shown by horizontal error bars. Symbols: open - ejection, solid - suction; dashed line - CFD simulation of ejection test, solid line - simulation of suction tests

Figure 6. Calibration plots $\left(C_{\mathrm{d}}\right.$ vs $\left.h_{0} / d_{\mathrm{t}}\right)$ for the liquids in Table 1 at a flow rate of $20 \mathrm{ml} / \mathrm{min}, 16.5{ }^{\circ} \mathrm{C}$. (a) ejection; (b) suction. Symbols: triangle $-18 \mathrm{wt} \%$ glycerol, $R e_{\mathrm{t}}=250$; diamond - paraffin oil, $R e_{\mathrm{t}}=0.36$; square - deionised water, $R e_{\mathrm{t}}=377$; circle $-8 \mathrm{wt} \%$ sucrose solution, $R e_{\mathrm{t}}=$ 349 ; cross $-10 \mathrm{wt} \%$ sucrose solution, $R e_{\mathrm{t}}=331$. Dashed line - CFD simulation, ejection: solid line - CFD simulation, suction (both for deionised water). Inserts show $C_{\mathrm{d}} v s h_{0} / d_{\mathrm{t}}$ in the usefully linear region

Figure 7. Effect of throat Reynolds number on $C_{\mathrm{d}}$ for deionised water (squares), $8 \%$ sucrose solution (triangles) and $10 \%$ sucrose solution (circles) in (i) ejection (open symbols) and (ii) suction (solid symbols) at $(a) h_{0} / d_{\mathrm{t}}=1.0,(b) h_{0} / d_{\mathrm{t}}=0.14,(c) h_{0} / d_{\mathrm{t}}=0.12$ and $(d) h_{0} / d_{\mathrm{t}}=0.10$

Figure 8. Comparison of experimental measurements, CFD simulations and Equations [6] and [10] for (a) $h_{0} / d_{\mathrm{t}}=0.14$ and (b) 0.10. Symbols, data: solid - this work, open - Tuladhar et al. [1]. Dashed line, Equation [6], solid lines, Equation [10], dotted lines - CFD simulation (deionized water). Gauging liquid: squares - deionised water, triangles - $8 \%$ sucrose solution, circles - $10 \%$ sucrose solution

Figure 9. Effect of surface roughness, expressed as $R_{\mathrm{a}}$, at $R e_{\mathrm{t}}=189$ (square), 283 (circle), 377 (diamond), 472 (triangle), 566 (cross), 660 (asterisk) and 754 (dash) at initial nozzle-substrate clearance (not corrected for roughness) $h / d_{\mathrm{t}}=0.10$. (a) ejection - black and $(b)$ suction - red. The surfaces are (from left to right) smooth, P240, P150, P180 and P120 (note the roughness of P150 is lower than P180 due to the poor quality control (Mell [9]). Representative uncertainty bars shown for one dataset

Figure 10. Calibration curves $\left(C_{\mathrm{d}}\right.$ vs $\left.h_{\text {true }} / d_{\mathrm{t}}\right)$ on smooth and rough surfaces (the latter have been corrected for measured roughness). Symbols (Table 2): diamond - P240, triangle - P180, square $-\mathrm{P} 150$ and circle $-\mathrm{P} 120$. Gauging conditions: deionised water $\left(16.5^{\circ} \mathrm{C}\right),(a) R e_{\mathrm{t}}=$ 
377 and $(b) R e_{\mathrm{t}}=754$; (i) ejection, (ii) Suction. Loci are interpolations of experimental data for smooth surfaces

\section{Text for table of contents}

A bench-top device that can be used to perform fluid dynamic gauging measurements of soft solid layers with zero net liquid discharge and potentially under aseptic conditions is demonstrated. Testing results are presented for Newtonian liquids with a range of viscosities: deionised water, sucrose solutions, glycerol/water solutions and a paraffin oil. The experimental data for discharge coefficient against clearance/nozzle throat diameter gave good agreement with CFD simulations. The influence of surface roughness was studied by making measurements on a series of commercial sandpapers. 
Schematic of ZFDG nozzle geometry. h0 is the clearance between the nozzle and the substrate. Dimensions: $\theta=45^{\circ}, \mathrm{dt}=1.0 \mathrm{~mm}, \mathrm{di}=5.9 \mathrm{~mm}, \mathrm{we}=0.15 \mathrm{~mm}, \mathrm{wr}=1.0 \mathrm{~mm}$. Dotted streamline indicates the flow path taken by the liquid in the ejection phase; the dashed streamline shows that in suction (with flow recirculation which occurs under certain operating conditions) $1921 \times 1515 \mathrm{~mm}(72 \times 72 \mathrm{DPI})$ 


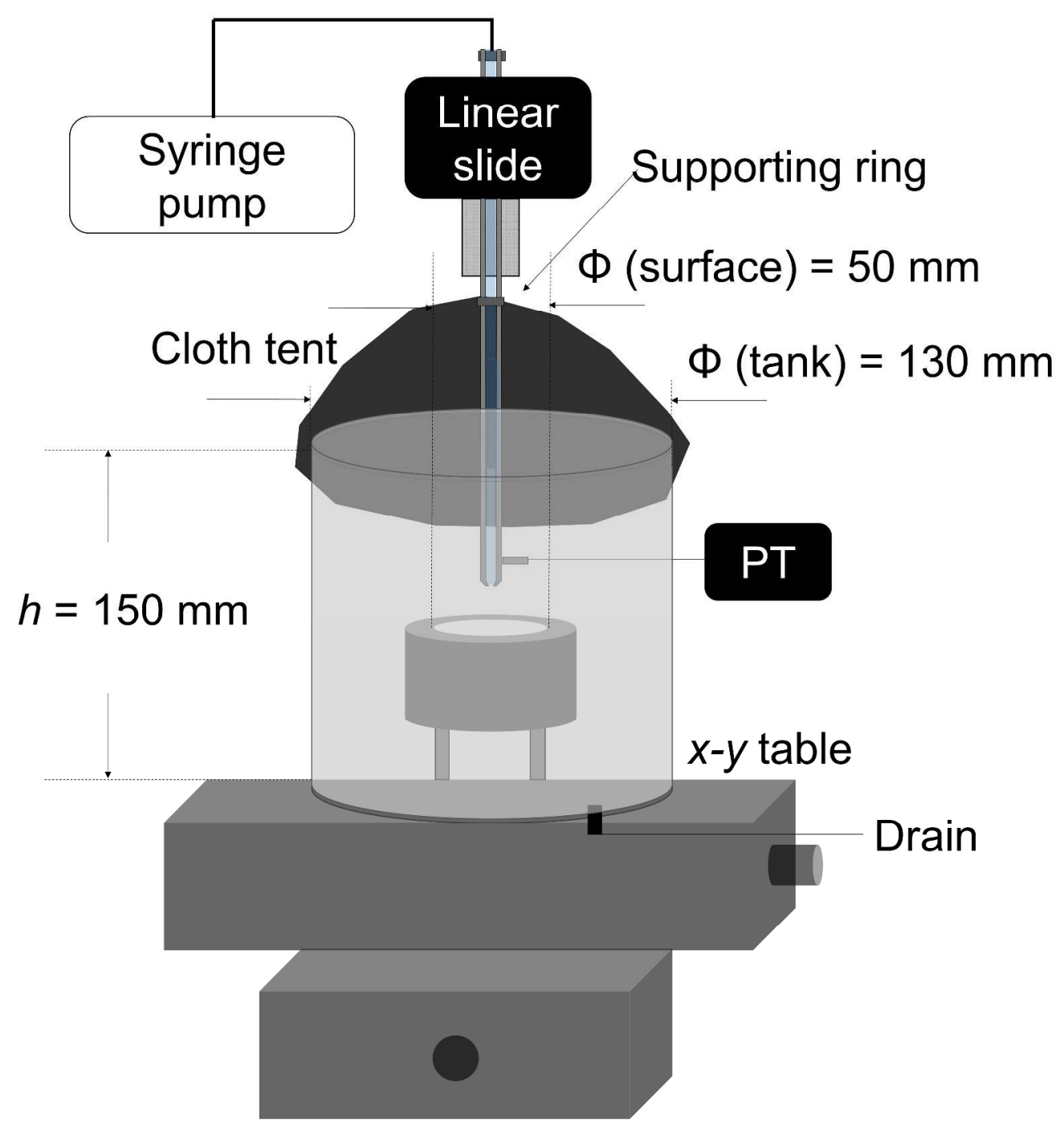

Schematic of aseptic ZFDG system $1405 \times 1509 \mathrm{~mm}$ ( $72 \times 72$ DPI) 
(a)

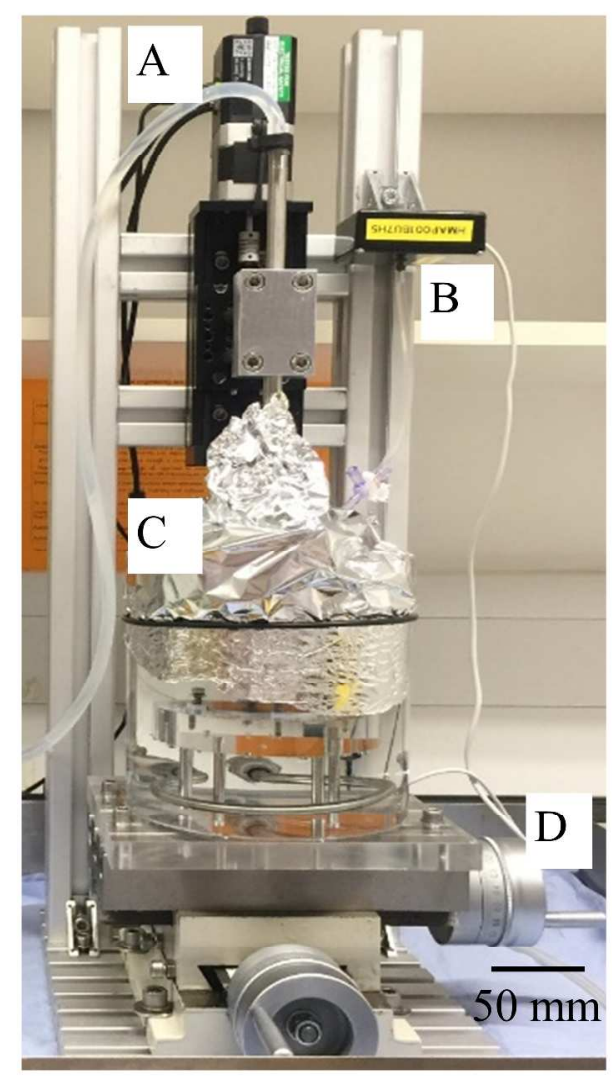

(b)

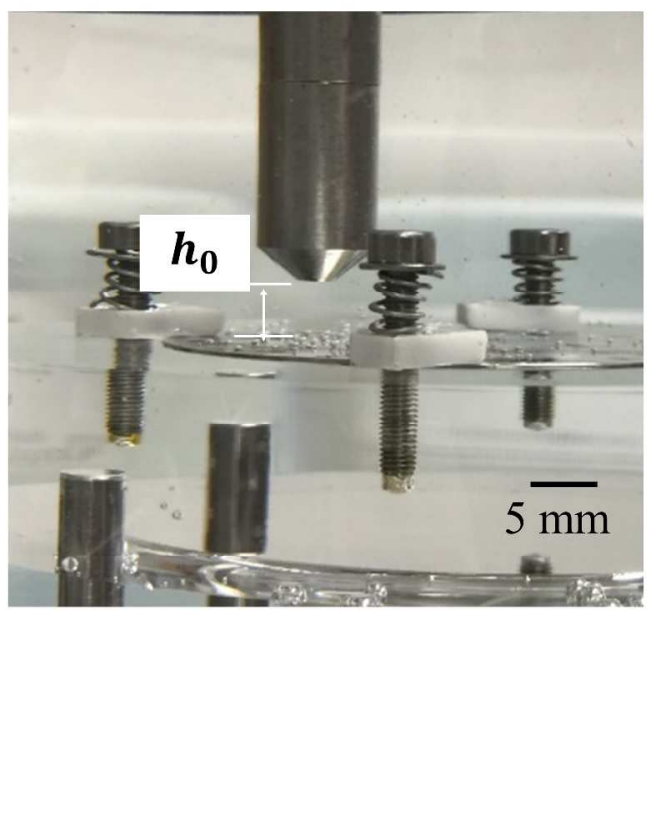

Photographs of ZFDG (a) test rig, and (b) detailed view of $1 \mathrm{~mm}$ i.d. nozzle and substrate. Labels: A, linear slide; $\mathrm{B}$, pressure transducer; $\mathrm{C}$, gauging tank; $\mathrm{D}, \mathrm{x}-\mathrm{y}$ table $1651 \times 1507 \mathrm{~mm}(72 \times 72 \mathrm{DPI})$ 
(a)

(b)

A

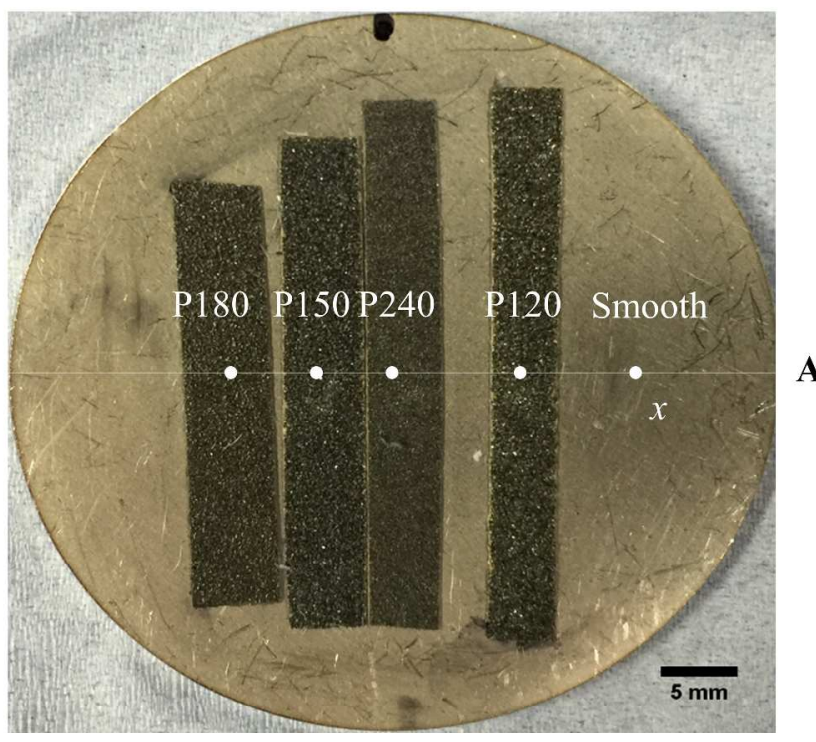

A

(c)
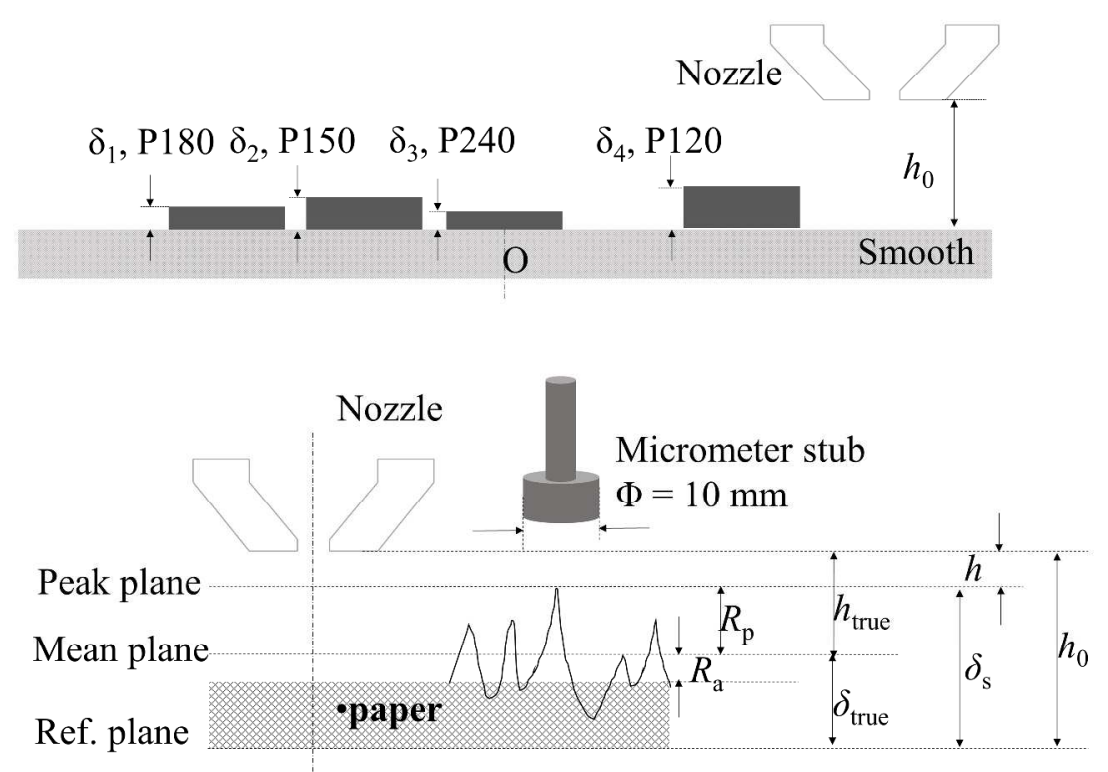

(a) Photograph, plan view of stainless steel disc with strips of sandpaper of different grit sizes attached. The gauging nozzle was moved across trajectory AA. Points indicate where gauging measurements were made;

(b) Side view (not to scale) schematic of sandpaper thicknesses for zeroing, $\delta 1-4$ - thicknesses of sandpaper (P180, P150, P240 and P120, respectively); (c) Schematic (not to scale) of rough surface: Rp is the average height between highest peak and mean plane, $\mathrm{Ra}$ is the mean roughness, $\delta \mathrm{s}$ is the thickness measured by micrometer, otrue the true thickness, htrue the corrected/true clearance from the nozzle to mean plane of sandpaper. The smooth substrate is the reference plane $1424 \times 1833 \mathrm{~mm}(72 \times 72 \mathrm{DPI})$ 


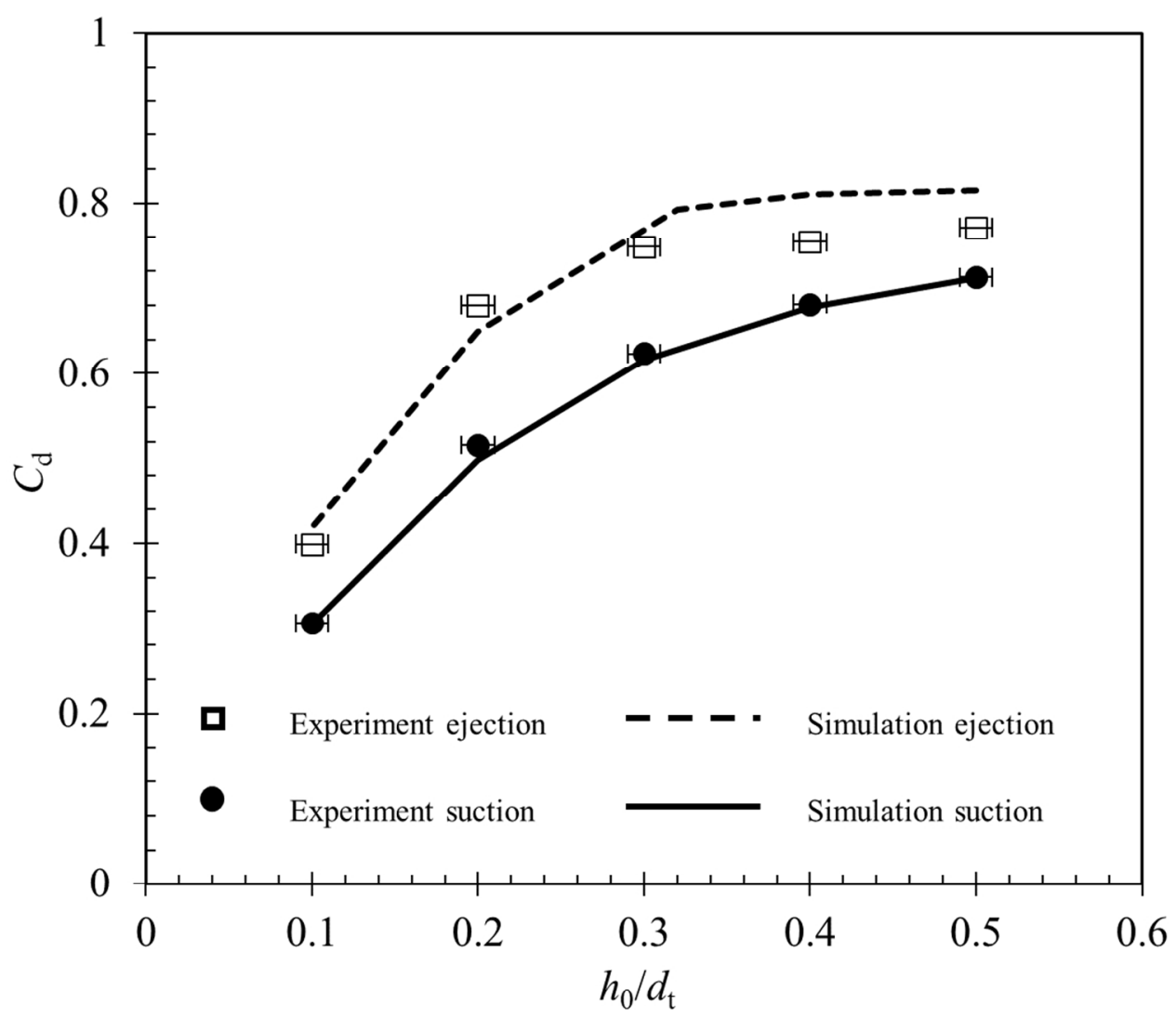

Reproducibility of ZFDG measurements on clean steel substrate. Deionised water at $16.5^{\circ} \mathrm{C}$, flow rate 20 $\mathrm{ml} / \mathrm{min}$. Ten data sets were collected at each $\mathrm{h} 0 / \mathrm{dt}$ value from 0.1 to 0.5 . The mean $\mathrm{Cd}$ value is plotted against ho/dt: the standard deviation is less than $0.2 \%$ of each datum (not shown), and uncertainty in $\mathrm{h}$ is shown by horizontal error bars. Symbols: open - ejection, solid - suction; dashed line - CFD simulation of ejection test, solid line - simulation of suction tests

$$
423 \times 359 \mathrm{~mm}(72 \times 72 \mathrm{DPI})
$$


Calibration plots (Cd vs ho/dt) for the liquids in Table 1 at a flow rate of $20 \mathrm{ml} / \mathrm{min}, 16.5^{\circ} \mathrm{C}$. (a) ejection; (b) suction. Symbols: triangle -18 wt $\%$ glycerol, Ret $=250$; diamond - paraffin oil, Ret $=0.36$; square deionised water, Ret $=377$; circle $-8 \mathrm{wt} \%$ sucrose solution, Ret $=349$; cross $-10 \mathrm{wt} \%$ sucrose solution, Ret $=331$. Dashed line - CFD simulation, ejection: solid line - CFD simulation, suction (both for deionised water). Inserts show $\mathrm{Cd}$ vs ho/dt in the usefully linear region $499 \times 793 \mathrm{~mm}(72 \times 72 \mathrm{DPI})$ 

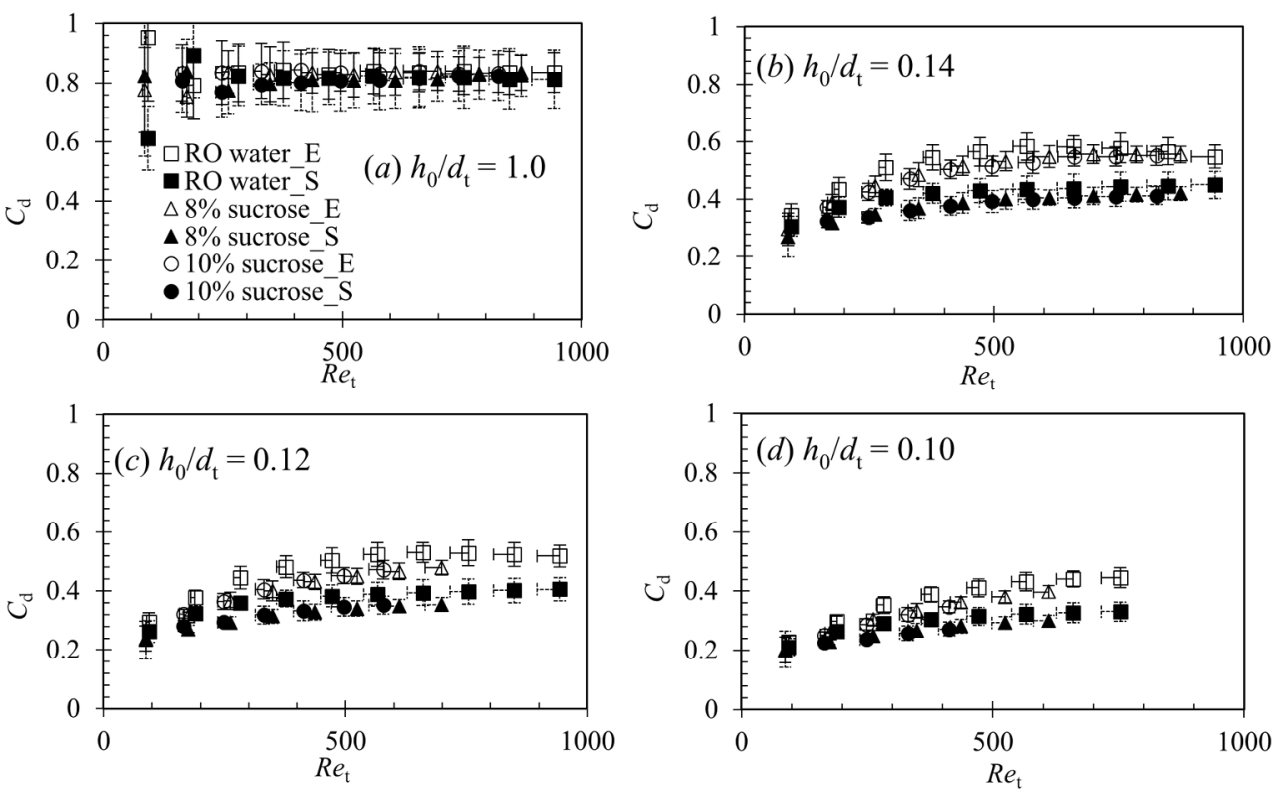

Effect of throat Reynolds number on Cd for deionised water (squares), $8 \%$ sucrose solution (triangles) and $10 \%$ sucrose solution (circles) in (i) ejection (open symbols) and (ii) suction (solid symbols) at (a) ho/dt = 1.0 , (b) ho/dt $=0.14$, (c) ho/dt $=0.12$ and (d) ho/dt $=0.10$ $696 \times 431 \mathrm{~mm}(72 \times 72 \mathrm{DPI})$ 

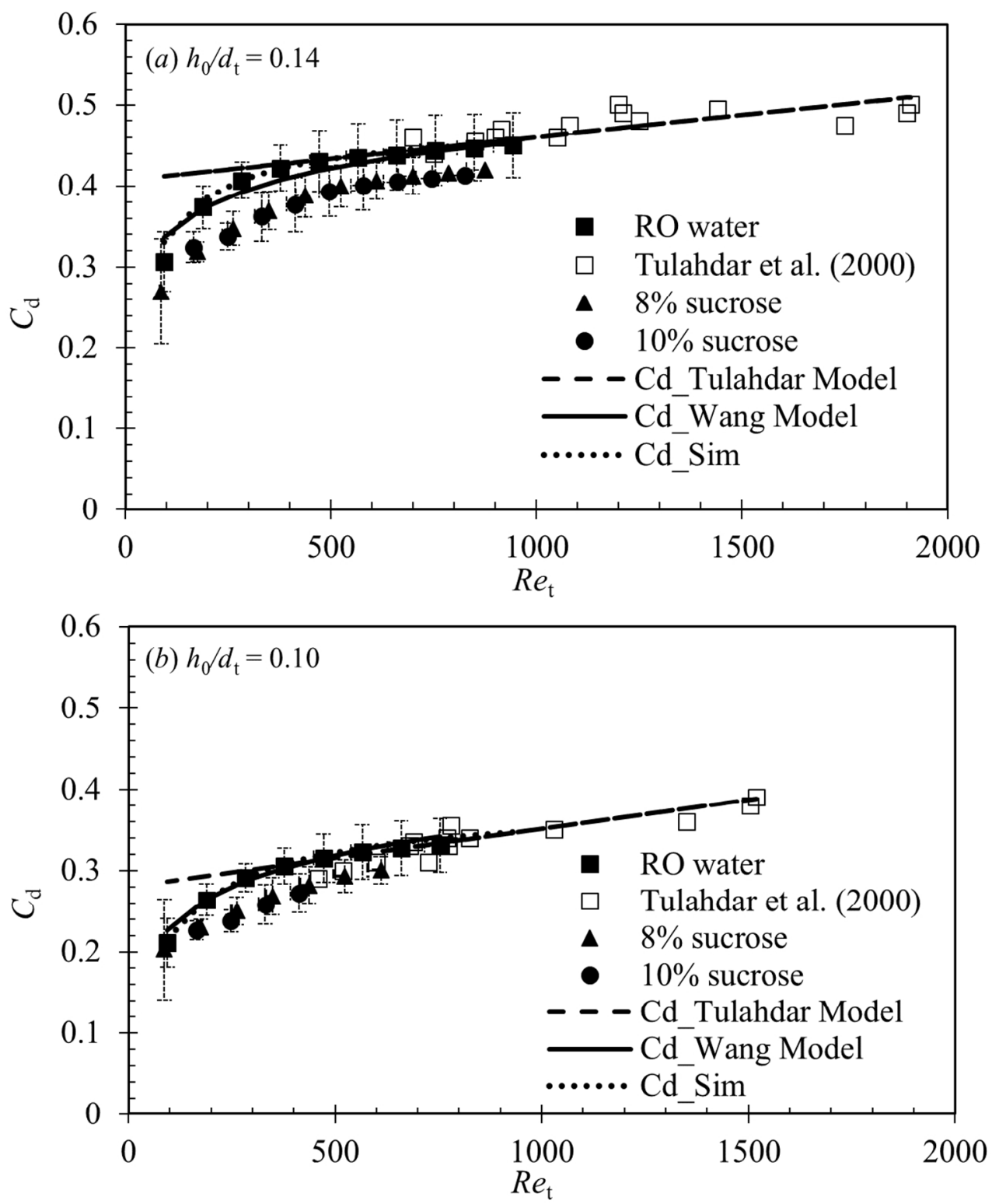

Comparison of experimental measurements, CFD simulations and Equations [6] and [10] for (a) h0/dt = 0.14 and (b) 0.10. Symbols, data: solid - this work, open - Tuladhar et al. [1]. Dashed line, Equation [6], solid lines, Equation [10], dotted lines - CFD simulation (deionized water). Gauging liquid: squares deionised water, triangles - $8 \%$ sucrose solution, circles - $10 \%$ sucrose solution $459 \times 552 \mathrm{~mm}$ (72 x 72 DPI) 

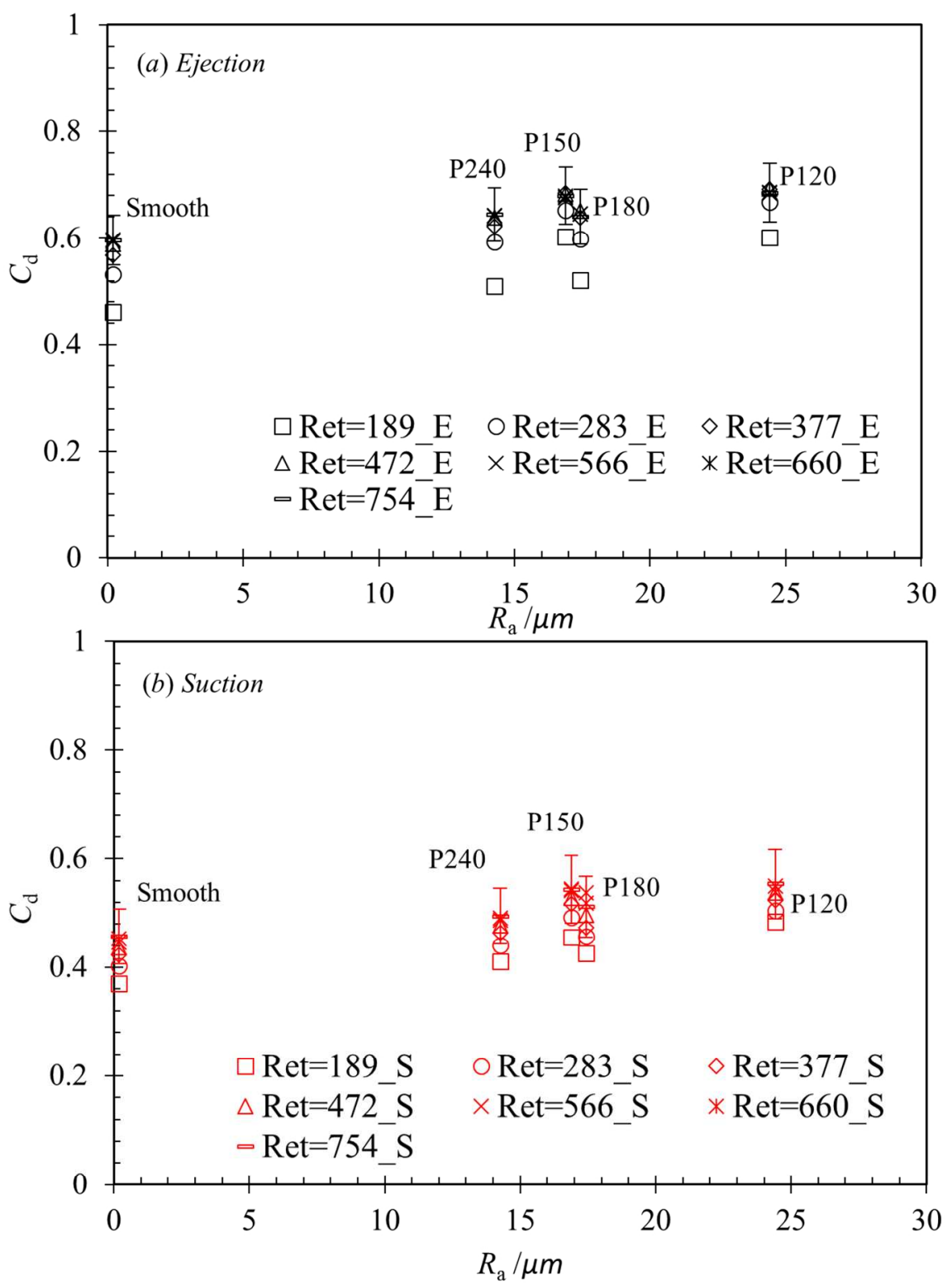

Effect of surface roughness, expressed as Ra, at Ret = 189 (square), 283 (circle), 377 (diamond), 472 (triangle), 566 (cross), 660 (asterisk) and 754 (dash) at initial nozzle-substrate clearance (not corrected for roughness) $\mathrm{h} / \mathrm{dt}=0.10$. (a) ejection - black and (b) suction - red. The surfaces are (from left to right) smooth, P240, P150, P180 and P120 (note the roughness of P150 is lower than P180 due to the poor quality control (Mell [9]). Representative uncertainty bars shown for one dataset $461 \times 615 \mathrm{~mm}(72 \times 72 \mathrm{DPI})$ 
(a)
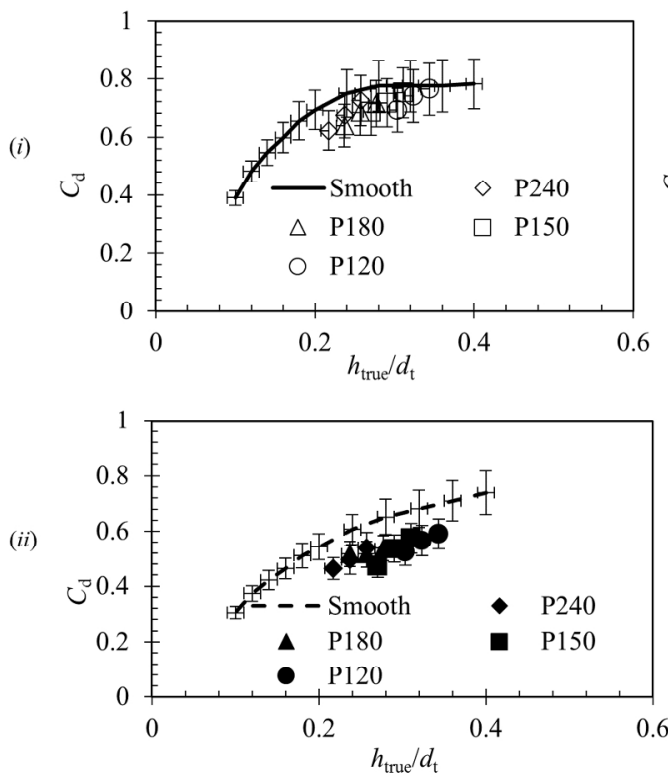

(b)
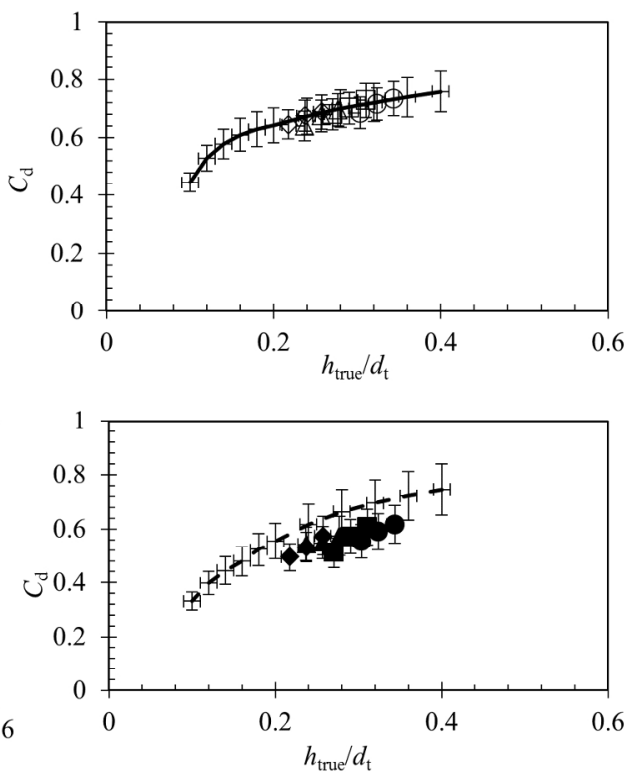

Calibration curves (Cd vs htrue/dt) on smooth and rough surfaces (the latter have been corrected for measured roughness). Symbols (Table 2): diamond - P240, triangle - P180, square - P150 and circle P120. Gauging conditions: deionised water $\left(16.5^{\circ} \mathrm{C}\right.$ ), (a) Ret $=377$ and (b) Ret $=754$; (i) ejection, (ii) Suction. Loci are interpolations of experimental data for smooth surfaces $715 \times 465 \mathrm{~mm}(72 \times 72 \mathrm{DPI})$ 
1

2

3

4

5

6

7

8

9

10

11

12

13

14

15

16

17

18

19

20

21

22

23

24

25

26

27

28

29

30

31

32

33

34

35

36

37

38

39

40

41

42

43

44

45

46

47

48

49

50

51

52

53

54

55

56

57

58

59

60
Table 1. Physical properties of gauging liquids $\left(16.5^{\circ} \mathrm{C}\right)$

\begin{tabular}{lccccc}
\hline Gauging liquid & $\rho$ & $\mu$ & $R e_{\mathrm{t}}(20 \mathrm{ml} / \mathrm{min})$ & Supplier & Symbol \\
& $\mathrm{kg} / \mathrm{m}^{3}$ & $\mathrm{mPa} . \mathrm{s}$ & - & & Figure 8 \\
\hline Deionised water & 997.3 & 1.12 & 377 & $\mathrm{~N} / \mathrm{A}$ & $\mathbf{\square}$ \\
$8 \mathrm{wt} \%$ sucrose & 1029.9 & 1.25 & 349 & Sigma & $\boldsymbol{\Delta}$ \\
$10 \mathrm{wt} \%$ surcrose & 1038.1 & 1.33 & 331 & Sigma & $\bullet$ \\
$18 \mathrm{wt} \%$ glycerol & 1047.6 & 1.65 & 250 & Aldrich & N/A \\
Paraffin oil & 860 & 1000 & 0.36 & Sigma-Aldrich & N/A \\
\hline
\end{tabular}


Table 2. Sandpaper surface roughness measures, see Figure 4(b): $R_{\mathrm{a}}$ (arithmetical mean height), $R_{\mathrm{z}}$ (average height between highest peak and deepest valley), $R_{\mathrm{p}}$ (average height between highest peak and mean plane), and average particle sizes

\begin{tabular}{|c|c|c|c|c|c|}
\hline \multirow[t]{2}{*}{ Surface } & $R_{\mathrm{a}}$ & $R_{\mathrm{z}}$ & $R_{\mathrm{p}}$ & Average particle diameter & Symbol \\
\hline & $\mu \mathrm{m}$ & $\mu \mathrm{m}$ & $\mathrm{mm}$ & $\mu \mathrm{m}$ & Figure 10 \\
\hline Smooth & 0.2 & 0.97 & 0.0007 & N/A & Lines \\
\hline $\mathrm{P} 240^{*}$ & $\sim 14.26$ & $\sim 169.6$ & 0.12 & 68 & $\diamond$ \\
\hline P180 & 17.43 & 198.1 & 0.14 & 82 & $\Delta$ \\
\hline $\mathrm{P} 150$ & 16.89 & 229.9 & 0.17 & $92-100$ & $\square$ \\
\hline $\mathrm{P} 120$ & 24.41 & 273.2 & 0.20 & $115-125$ & $\mathrm{O}$ \\
\hline
\end{tabular}

* the data are for P220 due to lack of data on P240 